\title{
LIFE CYCLE ASSESSMENT OF POINT-OF-LAY BIRDS TO FROZEN CHICKEN PRODUCTION IN A TROPICAL ENVIRONMENT
}

\author{
TEMITAYO A. EWEMOJE ${ }^{1 *}$, OLUFEMI P. ABIMBOLA ${ }^{1}$, OLAYINKA A. \\ OMOTOSHO $^{2}$ \\ ${ }^{1}$ Department of Agricultural \& Environmental Engineering Department, Faculty of \\ Technology, University of Ibadan, Ibadan \\ ${ }^{2}$ Agricultural \& Environmental Engineering Unit, Institute of Agricultural Research \& \\ Training, Obafemi Awolowo University, Moor Plantation, Ibadan, Oyo State, Nigeria
}

\begin{abstract}
The study examined four scenarios for reduction of environmental impacts with use of $100 \%$ purchased electricity in all processing activities as first scenario while second scenario was the use of $100 \%$ electricity from diesel generators. Third and fourth scenarios were to use $50 \%$ each of both purchased electricity and diesel-generated electricity in all activities. Most of the Energy Use (EU) came during the freezing process. Global Warming Potential (GWP) value for Scenario 2 is predominantly higher than the values for other three scenarios. The results show that the major source for global warming potential for Scenario 2 is the freezing process, whereas for the other three scenarios the animal management represents the main contributor. Similar to GWP, the Acidification Potential and Eutrophication Potential values for Scenario 2 were higher, although very small and this may be attributed to diesel generators emitting slightly higher amounts of $\mathrm{NO}_{\mathrm{x}}$ and $\mathrm{SO}_{\mathrm{x}}$.
\end{abstract}

Keywords: environmental impacts, poultry system, energy use scenarios, life cycle assessment

\section{INTRODUCTION}

Poultry production is one of the major contributors to global environmental degradation. Currently, livestock raised for meat uses about a third of global ice-free terrestrial land and produces $18 \%$ of global greenhouse gas (GHG) emissions.

This is more than the global transportation sector [1]. Livestock production is also one of the main drivers of deforestation and degradation of wildlife habitats. Due to increasing population size and per capita meat consumption in developing world, consumption of global meat is expected to double between 1999 and 2050 [1]. Such increases will also double the impacts of frozen chicken on the environment unless more efficient chicken production methods are adopted.

Policy makers are increasingly using environmental variables in decision-making and one of the ways to generate environmental information involves Life Cycle Assessment (LCA) methodology that measures the environmental impacts of a product throughout its life cycle [2].

\footnotetext{
"Corresponding author, email: tayo ewemoje@yahoo.co.uk

(C) 2017 Alma Mater Publishing House
} 
LCA has emerged as a standardized environmental management tool capable of analyzing environmental burdens along the supply chain of products and processes [3]. It is a methodology for examining environmental impacts associated with a product, process or service "from cradle to grave" [4] such as land or fossil fuels, and the emissions to the environment.

Feed production according to [5] and [6] has been identified as one of the major contributors to the environmental impacts (50-85\% for climate change, 64-97\% for eutrophication potential, 70-96\% for energy use) of animal production systems.

LCA allows a comprehensive view of the various impacts on the environment, enabling the identification of suitable measures from a sustainable development with the soaring worldwide demand to set reliable environmental criteria for food and feed products. This has brought LCA methodologies into agribusiness as a way to support the decision-making processes regarding agriculture and food production technologies $[7,8]$.

Furthermore, LCA is considered to be a tool that can be applied to evaluate agricultural production systems and it is based on an inventory of the resources consumed and the emissions to the environment at each stage of the product life cycle. Human health, natural resources and natural environment are classified as areas of protection in life cycle impact assessment (LCIA) by International Standards Organization [9].

Environmental impacts can be classified in many ways which relate to the scale of impacts, the timing of impacts, and the phase of production and the target of impact [10]. Impacts on the environment can be local, regional or global; it can be past, current and future [11]. Previous operations may lead to groundwater pollution on a long term future [10]. The authors [12] evaluated environmental impacts contributed by beef cow-calf system and the approaches to lower the impacts.

In agricultural production systems, other greenhouse gases (GHGs) such as $\mathrm{CO}_{2}, \mathrm{CH}_{4}$, in addition to $\mathrm{N}_{2} \mathrm{O}$ can be emitted [13]. Opining that a holistic approach is needed if overall impact of the agricultural production systems on global greenhouse emissions is to be addressed.

This have the advantage of identifying environmental impacts of all stages in the production cycle rather than focusing on a single source of GHG emission for comparative or improvement purposes [13], which is the primary purpose of this studies. Hence, the objectives of this study are: (I) to estimate the potential environmental impacts of large-scale poultry layer system from point-of-lay to frozen chicken; (II) to investigate the effects of four scenarios in processes from point-of-lay to frozen chicken.

\section{MATERIALS AND METHODS}

LCA methodology consists of four major stages: (I) Goal and scope definition, (II) Life cycle inventory, (III) Life cycle impact assessment and (IV) Interpretation of result. The functional unit (FU), towards which all the impacts are allocated, is defined as $1.35 \mathrm{~kg}$ of marketed frozen chicken. The system boundary, indicated by the dashed line in Figure 1 and Table 1, covers the major processing activities from point-of-lay to frozen chicken production.

Ammonia losses due to housing and manure storage are assumed to be respectively $25 \%$ and $10 \%$. This are based on the assumptions that (I) roofed housing facility was used for keeping layers for a calendar year and also for temporarily keeping spent layers for a day before slaughtering and (II) manure produced was temporarily stacked without turning [14]. This study considers emissions from wastes due to storage during laying period and one-day pre-slaughtering only. Emissions from solid wastes of frozen chicken processing (viscera, heads and feet) and inputs to waste treatment from processing of frozen chicken are not included in this study as solid wastes produced from these processes are sold out.

The period from point-of-lay to spent layer lasts for some weeks i.e. bird starts laying at the age of 18-20 weeks depending on genetic and environmental factors such as light and nutrition. Laying continues thereafter for a period of one calendar year. After the onset of laying, peak production is attained within 6 weeks. At this stage, the rate of lay will be greater than $80-90 \%$. This production level will continue and gradually decline at about 48 weeks until it is uneconomical to keep the birds. Breed of birds used in this study was the near black layers. 


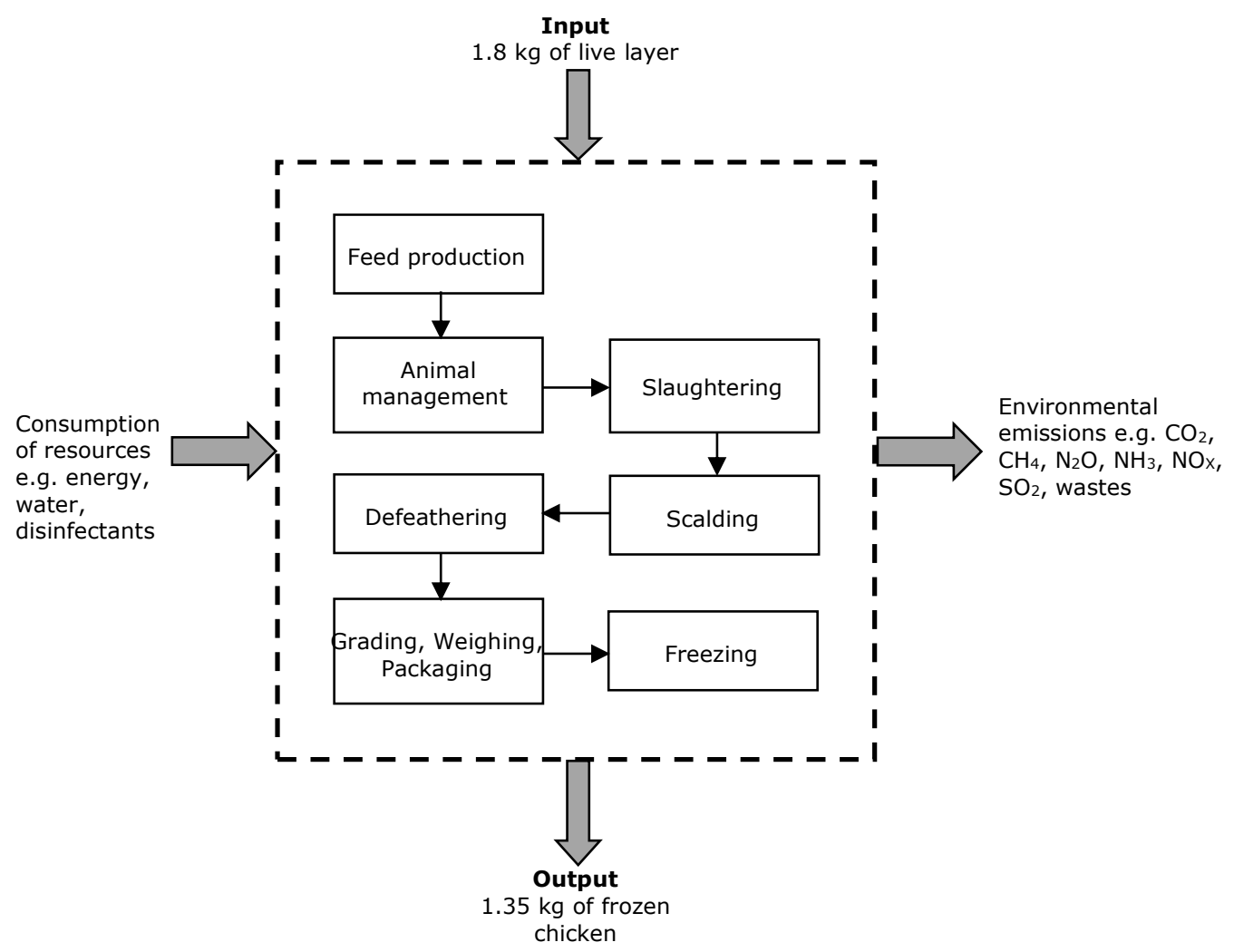

Fig. 1. System boundary of frozen chicken production in LCA study.

Table 1. Description of scenarios.

\begin{tabular}{|l|c|c|l|}
\hline \multirow{2}{*}{ Scenario } & \multicolumn{2}{|c|}{ Power source } & \multicolumn{2}{|c|}{ Other important features of scenario } \\
\cline { 2 - 3 } & $\begin{array}{c}\text { Purchased } \\
\text { electricity }\end{array}$ & $\begin{array}{c}\text { Diesel } \\
\text { generators }\end{array}$ & \\
\hline Scenario 1 & $100 \%$ & $0 \%$ & $\begin{array}{l}\text { Purchased electricity is based on 22.4 \% hydropower and } \\
77.6 \% \text { thermal power }\end{array}$ \\
\hline Scenario 2 & $0 \%$ & $100 \%$ & Electricity from diesel stand-by generators \\
\hline Scenario 3 & $50 \%$ & $50 \%$ & $\begin{array}{l}\text { Purchased electricity is based on 22.4 \% hydropower and } \\
77.6 \% \text { thermal power }\end{array}$ \\
\hline Scenario 4 & $50 \%$ & $50 \%$ & $\begin{array}{l}\text { Similar to scenario 3 except that purchased electricity } \\
\text { generation is based on 50 \% hydropower and 50 \% thermal } \\
\text { power }\end{array}$ \\
\hline
\end{tabular}

Roofed housing facility was used for keeping the layers for a year and also for temporary keeping of live spent layers for a day before slaughtering. The manure generated was temporarily stacked without turning. All activities from point-of-lay to spent layer were assumed to be carried out for 24 hours/day and 365 days a year. There is an exception for feed production and water pumping which are assumed to be for less than an hour per day.

All frozen chicken processing activities in this study were carried out for 7 hours/day, 6 days/week all year round. There was an exception for the blast cold rooms which were assumed to be in operation for 24 hours/day yearround. Live spent layers with an average live weight of $1.8 \mathrm{~kg}$ were slaughtered every day for processing. Slaughtering was the only frozen chicken processing activity done manually and not accounted for in this study. It was assumed that each pack of chicken stayed for a day in the blast cold rooms before it was taken to the gate (i.e. sold at gate) and that diesel was used in the transportation of birds and feed.

\subsection{Life cycle inventory and assessment factors}

An inventory of all the resources used and all the emissions released into the environment was done at the second stage of LCA. This covers all the activities within the system boundary from point-of-lay to frozen chicken 
production. Lighting the poultry houses, preparation of feed, transportation of birds and feed and carrying manure out of the housing facility were considered as work associated with animal management.

Emission factors for the consumption of purchased power accounted for emissions from hydropower generation as well as the relatively large proportions of emissions from natural gas combustion at thermal gas stations. Table 2 showed environmental loads emission factors and these emission factors do not include emissions associated with construction of reservoirs, extraction, production and transportation of burned fuel to produce electricity.

Primary energy conversion factors and GHG emission factors for diesel and natural gas were based on [15] which uses U.S. EPA's Emissions and Generation Resource Integrated Database's (eGRID). This study assumed that hydropower generation, on the average, emits one-thirty-fifth of the GHG [15]. Table 3 showed environmental loads emission factors for diesel. Pollutants emitted were also determined using [16].

Table 2. Environmental loads associated with frozen chicken production and purchased electricity emission factors.

\begin{tabular}{|c|c|c|c|c|}
\hline Emissions & \multicolumn{2}{|c|}{ Natural gas factor } & Hydropower factor & $\begin{array}{c}\text { Equivalent purchased } \\
\text { electricity factor }\end{array}$ \\
\hline units & $\begin{array}{c}\text { lbs per } \\
\text { million Btu }\end{array}$ & kg per kWh & kg per kWh & $\mathrm{kg}$ per kWh \\
\hline $\mathrm{CO}_{2}$ & 117.6 & $1.56338 \mathrm{E}-08$ & $1.15806 \mathrm{E}-10$ & $1.21577 \mathrm{E}-08$ \\
\hline $\mathrm{CH}_{4}$ & 0.0225 & $2.99115 \mathrm{E}-12$ & $2.21567 \mathrm{E}-14$ & $2.3261 \mathrm{E}-12$ \\
\hline $\mathrm{N}_{2} \mathrm{O}$ & 0.0022 & $2.92468 \mathrm{E}-13$ & $2.16643 \mathrm{E}-15$ & $2.27441 \mathrm{E}-13$ \\
\hline $\mathrm{SO}_{2}$ & 0.0006 & $7.97641 \mathrm{E}-14$ & 0 & $6.1897 \mathrm{E}-14$ \\
\hline $\mathrm{NO}_{\mathrm{X}}$ & 0.098 & $1.30281 \mathrm{E}-11$ & 0 & $1.01098 \mathrm{E}-11$ \\
\hline
\end{tabular}

Table 3. Environmental loads associated with frozen chicken production and diesel emission factors.

\begin{tabular}{|c|c|c|}
\hline Emissions & Diesel emission factor & Diesel emission factor \\
\hline Units & lbs./gallon & $\mathrm{kg} / \mathrm{liter}$ \\
\hline $\mathrm{CO}_{2}$ & 25 & 2.145232732 \\
$\mathrm{CH}_{4}$ & 0.000216 & 0.000177605 \\
$\mathrm{~N}_{2} \mathrm{O}$ & 0.00011 & $4.98891 \mathrm{E}-06$ \\
$\mathrm{SO}_{2}$ & 0.15 & 0.014168514 \\
$\mathrm{NO}_{\mathrm{X}}$ & 0.02 & 0.001796009 \\
\hline
\end{tabular}

All electricity emission factors were multiplied by the power ratings of equipment used in each processing activity while the loading rate and power factor of the diesel generators, together with the power ratings of equipment, were multiplied with the diesel emission factors in computing the emissions in the activities.

The global warming potential, an index for estimating the global warming contribution due to atmospheric emission of GHGs, was calculated using equation (1) and the $\mathrm{CO}_{2}$-equivalent factors by Intergovernmental Panel on Climate Change [17] for $\mathrm{CO}_{2}: 1, \mathrm{CH}_{4}: 25$ and $\mathrm{N}_{2} \mathrm{O}: 298$.

These factors were set based on a time horizon of 100 years. The $\mathrm{SO}_{2}$ - equivalent factors for $\mathrm{SO}_{2}: 1, \mathrm{NO}_{\mathrm{X}}: 0.7$ and $\mathrm{NH}_{3}: 1.88$, derived from [18] and the $\mathrm{PO}_{4}$ - equivalent factors for $\mathrm{NO}_{\mathrm{X}}: 0.13$ and $\mathrm{NH}_{3}: 0.33$, derived from [19] were used in calculating the acidification and eutrophication potentials respectively with equations (2) and (3).

Mass of $\mathrm{CO}_{2}$-equivalent $=($ Mass of gas in $\mathrm{kg}) \times($ global warming potential $)$

Mass of $\mathrm{SO}_{2}-$ equivalent $=($ Mass of gas in $\mathrm{kg}) \times($ acidification potential $)$

Mass of $\mathrm{PO}_{4}-$ equivalent $=($ Mass of gas in $\mathrm{kg}) \times($ eutrophication potential $)$ 


\section{RESULTS AND DISCUSSION}

The effects of each process in the frozen chicken production on the environment based on the energy requirements of the equipment and their times of operation were examined. Total energy use and environmental impacts of producing $1.35 \mathrm{~kg}$ frozen chicken for four scenarios are presented from Table 4 to Table 7.

\subsection{Impact assessment and scenario analysis}

In this study, four scenarios were examined for reduction of environmental impacts as presented in Table 1. The first scenario was the use of $100 \%$ purchased electricity in all processing activities. The purchased electricity from the national grid was generated from hydropower and thermal power (using natural gas as fuel) in the ratio 22.4:77.6.

Second scenario was the use of $100 \%$ electricity from diesel generators in all processing activities from point-oflay to spent layer. The third and fourth scenarios were to use $50 \%$ each of both purchased electricity and dieselgenerated electricity in all activities but with different ratios of hydropower to thermal power in the purchased electricity as shown in Table 1 . The aim of the scenarios is to determine if there are significant changes in environmental impact loads.

\subsubsection{Global Warming Potential}

The global warming potential value for Scenario 2 (100\% Diesel-generated electricity) is predominantly higher than the values for other three scenarios (Table 8). The reason behind this is that there are more emissions from the combustion of diesel than for using either purchased power solely or combining it with diesel in any proportion. This is as a result of the comparatively smaller emission factors for purchased electricity, which is a national power mix of hydroelectricity and thermal electricity.

Table 5 shows that the major source for global warming potential for Scenario 2 was freezing which accounted for almost $90 \%$ of the total contribution. This was mostly due to 24 hour/day operation of the blast freezers. Scalding accounted for only about $6 \%$ of the total contribution. Animal management accounted for almost all the GHG emissions in the other three scenarios (Tables 4, 6 and 7) and was majorly due to the $\mathrm{CO}_{2}$ emissions from diesel used in transportation.

Table 4. Global warming potential (GWP), acidification potential (AP) and eutrophication potential (EP) values for each activity in scenario 1 .

\begin{tabular}{|c|c|c|c|c|c|c|c|c|c|}
\hline & Emission & $\begin{array}{c}\text { Animal } \\
\text { management }\end{array}$ & $\begin{array}{c}\text { Water } \\
\text { pumping }\end{array}$ & Scalding & $\begin{array}{c}\text { De- } \\
\text { feathering }\end{array}$ & Cutting & Packaging & Freezing & $\begin{array}{c}\text { Feed } \\
\text { production }\end{array}$ \\
\hline \multirow{5}{*}{ jWP } & $\mathrm{CO}_{2}$ & 6.67E-03 & $8.45 \mathrm{E}-12$ & $2.55 \mathrm{E}-10$ & $6.35 \mathrm{E}-11$ & $6.35 \mathrm{E}-11$ & $4.86 \mathrm{E}-11$ & 3.92E-09 & $3.54 \mathrm{E}-09$ \\
\hline & $\mathrm{CH}_{4}$ & $1.44 \mathrm{E}-06$ & $4.04 \mathrm{E}-14$ & $1.22 \mathrm{E}-12$ & 3.04E-13 & 3.04E-13 & 2.33E-13 & $1.87 \mathrm{E}-11$ & $1.69 \mathrm{E}-11$ \\
\hline & $\mathrm{N}_{2} \mathrm{O}$ & 8.74E-06 & $4.71 \mathrm{E}-14$ & $1.42 \mathrm{E}-12$ & $3.54 \mathrm{E}-13$ & $3.54 \mathrm{E}-13$ & $2.71 \mathrm{E}-13$ & $2.18 \mathrm{E}-11$ & $1.97 \mathrm{E}-11$ \\
\hline & Total & 6.68E-03 & $8.53 \mathrm{E}-12$ & $2.58 \mathrm{E}-10$ & $6.41 \mathrm{E}-11$ & $6.41 \mathrm{E}-11$ & $4.91 \mathrm{E}-11$ & 3.96E-09 & 3.57E-09 \\
\hline & $\begin{array}{l}\% \text { of Grand } \\
\text { total }\end{array}$ & 99.9999 & $1.28 \mathrm{E}-07$ & $3.86 \mathrm{E}-06$ & $9.61 \mathrm{E}-07$ & $9.61 \mathrm{E}-07$ & $7.36 \mathrm{E}-07$ & $5.93 \mathrm{E}-05$ & $5.36 \mathrm{E}-05$ \\
\hline \multirow{5}{*}{ AP } & $\mathrm{SO}_{2}$ & $4.00 \mathrm{E}-05$ & $4.30 \mathrm{E}-17$ & $1.30 \mathrm{E}-15$ & 3.23E-16 & 3.23E-16 & $2.48 \mathrm{E}-16$ & $1.99 \mathrm{E}-14$ & $1.80 \mathrm{E}-14$ \\
\hline & $\mathrm{NO}_{\mathrm{X}}$ & $3.73 \mathrm{E}-06$ & $4.92 \mathrm{E}-15$ & $1.49 \mathrm{E}-13$ & 3.69E-14 & 3.69E-14 & $2.83 \mathrm{E}-14$ & $2.28 \mathrm{E}-12$ & $2.06 \mathrm{E}-12$ \\
\hline & $\mathrm{NH}_{3}$ & 7.78E-01 & 0 & 0 & 0 & 0 & 0 & 0 & 0 \\
\hline & Total & 7.78E-01 & $4.96 \mathrm{E}-15$ & $1.50 \mathrm{E}-13$ & 3.73E-14 & 3.73E-14 & $2.86 \mathrm{E}-14$ & $2.30 \mathrm{E}-12$ & $2.08 \mathrm{E}-12$ \\
\hline & $\begin{array}{l}\% \text { of Grand } \\
\text { total }\end{array}$ & 100 & $6.37 \mathrm{E}-13$ & $1.93 \mathrm{E}-11$ & 4.79E-12 & $4.79 \mathrm{E}-12$ & 3.67E-12 & $2.95 \mathrm{E}-10$ & $2.67 \mathrm{E}-10$ \\
\hline \multirow{4}{*}{$\mathrm{EP}$} & $\mathrm{NO}_{\mathrm{X}}$ & 6.93E-07 & $9.13 \mathrm{E}-16$ & $2.76 \mathrm{E}-14$ & $6.86 \mathrm{E}-15$ & $6.86 \mathrm{E}-15$ & $5.26 \mathrm{E}-15$ & $4.23 \mathrm{E}-13$ & 3.82E-13 \\
\hline & $\mathrm{NH}_{3}$ & $1.37 \mathrm{E}-01$ & 0 & 0 & 0 & 0 & 0 & 0 & 0 \\
\hline & Total & $1.37 \mathrm{E}-01$ & $9.13 \mathrm{E}-16$ & $2.76 \mathrm{E}-14$ & $6.86 \mathrm{E}-15$ & $6.86 \mathrm{E}-15$ & $5.26 \mathrm{E}-15$ & $4.23 \mathrm{E}-13$ & $3.82 \mathrm{E}-13$ \\
\hline & $\begin{array}{l}\% \text { of Grand } \\
\text { total }\end{array}$ & 100 & $6.68 \mathrm{E}-13$ & $2.02 \mathrm{E}-11$ & $5.02 \mathrm{E}-12$ & $5.02 \mathrm{E}-12$ & $3.85 \mathrm{E}-12$ & $3.10 \mathrm{E}-10$ & $2.80 \mathrm{E}-10$ \\
\hline $\mathrm{EU}$ & & 12.5416 & 0.28774 & 75.6 & 18.792 & 18.792 & 14.4 & 1159.704 & 2.870235 \\
\hline
\end{tabular}

Units: GWP - $\mathrm{kg} \mathrm{CO}_{2}$ equivalent; $\mathrm{AP}-\mathrm{kg} \mathrm{SO}_{2}$ equivalent; $\mathrm{EP}-\mathrm{kg} \mathrm{PO} 4$ equivalent; $\mathrm{EU}$ - GJ/FU. All values are for a functional unit. 
Table 5. Global warming potential (GWP), acidification potential (AP) and eutrophication potential (EP) values for each activity in scenario 2 .

\begin{tabular}{|c|c|c|c|c|c|c|c|c|c|}
\hline & Emission & $\begin{array}{c}\text { Animal } \\
\text { manageme } \\
\text { nt }\end{array}$ & $\begin{array}{c}\text { Water } \\
\text { pumping }\end{array}$ & Scalding & $\begin{array}{c}\text { De- } \\
\text { feathering }\end{array}$ & Cutting & Packaging & Freezing & $\begin{array}{c}\text { Feed } \\
\text { production }\end{array}$ \\
\hline \multirow{5}{*}{ GWP } & $\mathrm{CO}_{2}$ & 9.72E-04 & $7.02 \mathrm{E}-05$ & $1.38 \mathrm{E}-02$ & $3.42 \mathrm{E}-03$ & $3.42 \mathrm{E}-03$ & $2.61 \mathrm{E}-03$ & $2.11 \mathrm{E}-01$ & $1.40 \mathrm{E}-04$ \\
\hline & $\mathrm{CH}_{4}$ & $2.10 \mathrm{E}-07$ & $1.52 \mathrm{E}-08$ & $2.97 \mathrm{E}-06$ & 7.38E-07 & 7.38E-07 & $5.64 \mathrm{E}-07$ & $4.56 \mathrm{E}-05$ & $3.02 \mathrm{E}-08$ \\
\hline & $\mathrm{N}_{2} \mathrm{O}$ & $1.27 \mathrm{E}-06$ & $9.20 \mathrm{E}-08$ & $1.80 \mathrm{E}-05$ & 4.48E-06 & $4.48 \mathrm{E}-06$ & $3.43 \mathrm{E}-06$ & $2.77 \mathrm{E}-04$ & $1.83 \mathrm{E}-07$ \\
\hline & Total & 9.73E-04 & 7.03E-05 & $1.38 \mathrm{E}-02$ & $3.42 \mathrm{E}-03$ & $3.42 \mathrm{E}-03$ & $2.62 \mathrm{E}-03$ & $2.11 \mathrm{E}-01$ & $1.40 \mathrm{E}-04$ \\
\hline & $\begin{array}{l}\% \text { of } \\
\text { Grand } \\
\text { total }\end{array}$ & 0.41305 & 0.02981 & 5.84344 & 1.45249 & 1.45249 & 1.11025 & 89.6391 & 0.05939 \\
\hline \multirow{5}{*}{ AP } & $\mathrm{SO}_{2}$ & $5.83 \mathrm{E}-06$ & 4.21E-07 & $8.25 \mathrm{E}-05$ & $2.05 \mathrm{E}-05$ & $2.05 \mathrm{E}-05$ & $1.57 \mathrm{E}-05$ & $1.27 \mathrm{E}-03$ & 8.39E-07 \\
\hline & $\mathrm{NO}_{\mathrm{X}}$ & $5.44 \mathrm{E}-07$ & $3.93 \mathrm{E}-08$ & 7.70E-06 & $1.91 \mathrm{E}-06$ & $1.91 \mathrm{E}-06$ & $1.46 \mathrm{E}-06$ & $1.18 \mathrm{E}-04$ & 7.83E-08 \\
\hline & $\mathrm{NH}_{3}$ & 7.78E-01 & 0 & 0 & 0 & 0 & 0 & 0 & 0 \\
\hline & Total & 7.78E-01 & $4.60 \mathrm{E}-07$ & $9.02 \mathrm{E}-05$ & $2.24 \mathrm{E}-05$ & $2.24 \mathrm{E}-05$ & $1.71 \mathrm{E}-05$ & $1.38 \mathrm{E}-03$ & $9.17 \mathrm{E}-07$ \\
\hline & $\begin{array}{l}\% \text { of } \\
\text { Grand } \\
\text { total }\end{array}$ & 99.8029 & 0.000059 & 0.01157 & 0.00288 & 0.00288 & 0.00220 & 0.17746 & 0.00012 \\
\hline \multirow{4}{*}{$\mathrm{EP}$} & $\mathrm{NO}_{\mathrm{X}}$ & $2.57 \mathrm{E}-07$ & $1.85 \mathrm{E}-08$ & $3.63 \mathrm{E}-06$ & $9.02 \mathrm{E}-07$ & $9.02 \mathrm{E}-07$ & $6.90 \mathrm{E}-07$ & $5.57 \mathrm{E}-05$ & 3.69E-08 \\
\hline & $\mathrm{NH}_{3}$ & $1.37 \mathrm{E}-01$ & 0 & 0 & 0 & 0 & 0 & 0 & 0 \\
\hline & Total & $1.37 \mathrm{E}-01$ & $1.85 \mathrm{E}-08$ & $3.63 \mathrm{E}-06$ & $9.02 \mathrm{E}-07$ & $9.02 \mathrm{E}-07$ & $6.90 \mathrm{E}-07$ & $5.57 \mathrm{E}-05$ & 3.69E-08 \\
\hline & $\begin{array}{l}\% \text { of } \\
\text { Grand } \\
\text { total }\end{array}$ & 99.9547 & 0.000014 & 0.00266 & 0.00066 & 0.00066 & 0.00050 & 0.04075 & 0.000027 \\
\hline EU & & 12.5416 & 0.90523 & 177.426 & 44.1023 & 44.1023 & 33.7110 & 2721.74 & 1.80332 \\
\hline
\end{tabular}

Units: GWP - $\mathrm{kg} \mathrm{CO}_{2}$ equivalent; $\mathrm{AP}-\mathrm{kg} \mathrm{SO}_{2}$ equivalent; $\mathrm{EP}-\mathrm{kg} \mathrm{PO}_{4}$ equivalent; $\mathrm{EU}-\mathrm{GJ} / \mathrm{FU}$. All values are for a functional unit.

Table 6. Global warming potential (GWP), acidification potential (AP) and eutrophication potential (EP) values for each activity in scenario 3 .

\begin{tabular}{|c|c|c|c|c|c|c|c|c|c|}
\hline & Emission & $\begin{array}{c}\text { Animal } \\
\text { management }\end{array}$ & $\begin{array}{c}\text { Water } \\
\text { pumping }\end{array}$ & Scalding & \begin{tabular}{|c|}
$\begin{array}{c}\text { Defeatheri } \\
\text { ng }\end{array}$ \\
\end{tabular} & Cutting & Packaging & Freezing & $\begin{array}{c}\text { Feed } \\
\text { production }\end{array}$ \\
\hline \multirow{5}{*}{ GWP } & $\mathrm{CO}_{2}$ & $6.67 \mathrm{E}-03$ & $1.25 \mathrm{E}-11$ & $3.79 \mathrm{E}-10$ & 9.43E-11 & 9.43E-11 & 7.22E-11 & 5.82E-09 & $5.26 \mathrm{E}-09$ \\
\hline & $\mathrm{CH}_{4}$ & $1.44 \mathrm{E}-06$ & $2.20 \mathrm{E}-14$ & $6.65 \mathrm{E}-13$ & $1.65 \mathrm{E}-13$ & $1.65 \mathrm{E}-13$ & $1.27 \mathrm{E}-13$ & $1.02 \mathrm{E}-11$ & 9.22E-12 \\
\hline & $\mathrm{N}_{2} \mathrm{O}$ & $8.74 \mathrm{E}-06$ & $3.45 \mathrm{E}-14$ & $1.04 \mathrm{E}-12$ & $2.59 \mathrm{E}-13$ & $2.59 \mathrm{E}-13$ & $1.98 \mathrm{E}-13$ & $1.60 \mathrm{E}-11$ & $1.44 \mathrm{E}-11$ \\
\hline & Total & $6.68 \mathrm{E}-03$ & $1.26 \mathrm{E}-11$ & $3.81 \mathrm{E}-10$ & 9.47E-11 & 9.47E-11 & 7.26E-11 & 5.84E-09 & 5.28E-09 \\
\hline & $\begin{array}{l}\% \text { of Grand } \\
\text { total }\end{array}$ & 99.9998 & $1.89 \mathrm{E}-07$ & $5.71 \mathrm{E}-06$ & $1.42 \mathrm{E}-06$ & $1.42 \mathrm{E}-06$ & $1.09 \mathrm{E}-06$ & $8.76 \mathrm{E}-05$ & $7.91 \mathrm{E}-05$ \\
\hline \multirow{5}{*}{ AP } & $\mathrm{SO}_{2}$ & T.00L-0J & $5.00 \mathrm{E}-14$ & $1.51 \mathrm{E}-12$ & $3.75 \mathrm{E}-13$ & $3.75 \mathrm{E}-13$ & $2.88 \mathrm{E}-13$ & -11 & $2.09 \mathrm{~L}-11$ \\
\hline & $\mathrm{NO}_{\mathrm{X}}$ & $3.73 \mathrm{E}-06$ & $7.12 \mathrm{E}-15$ & $2.15 \mathrm{E}-13$ & $5.35 \mathrm{E}-14$ & $5.35 \mathrm{E}-14$ & 4.10E-14 & 3.30 & $2.98 \mathrm{E}-12$ \\
\hline & $\mathrm{NH}_{3}$ & 7.78E-01 & 0 & 0 & 0 & 0 & 0 & 0 & 0 \\
\hline & \begin{tabular}{|l|} 
Total \\
\end{tabular} & 7.78E-01 & $5.71 \mathrm{E}-14$ & $1.73 \mathrm{E}-12$ & $4.29 \mathrm{E}-13$ & $4.29 \mathrm{E}-13$ & 3.29E-13 & $2.65 \mathrm{E}-11$ & 2.39E-11 \\
\hline & $\begin{array}{l}\% \text { of Grand } \\
\text { total }\end{array}$ & 99.9999 & $7.33 \mathrm{E}-12$ & $2.22 \mathrm{E}-10$ & $5.51 \mathrm{E}-11$ & $5.51 \mathrm{E}-11$ & $4.22 \mathrm{E}-11$ & 3.40 & $3.07 \mathrm{E}-09$ \\
\hline \multirow{4}{*}{ EP } & $\mathrm{NO}_{\mathrm{X}}$ & $6.93 \mathrm{E}-07$ & $1.32 \mathrm{E}-15$ & $4.00 \mathrm{E}-14$ & $9.93 \mathrm{E}-15$ & $9.93 \mathrm{E}-15$ & 7.61E-15 & $6.13 \mathrm{E}-13$ & $5.54 \mathrm{E}-13$ \\
\hline & $\mathrm{NH}_{3}$ & 1.37E-01 & 0 & 0 & 0 & 0 & 0 & 0 & 0 \\
\hline & Total & $1.37 \mathrm{E}-01$ & $1.32 \mathrm{E}-15$ & $4.00 \mathrm{E}-14$ & 9.93E-15 & 9.93E-15 & 7.61E-15 & $6.13 \mathrm{E}-13$ & $5.54 \mathrm{E}-13$ \\
\hline & $\begin{array}{l}\% \text { of Grand } \\
\text { total }\end{array}$ & 100 & $9.68 \mathrm{E}-13$ & 2.93E-11 & $7.27 \mathrm{E}-12$ & $7.27 \mathrm{E}-12$ & $5.57 \mathrm{E}-12$ & $4.49 \mathrm{E}-10$ & $4.05 \mathrm{E}-10$ \\
\hline $\mathrm{EU}$ & & 12.5416 & 0.59649 & 126.516 & 31.4471 & 31.4471 & 24.0555 & 1940.723 & 2.33678 \\
\hline
\end{tabular}


Units: GWP - $\mathrm{kg} \mathrm{CO}_{2}$ equivalent; $\mathrm{AP}-\mathrm{kg} \mathrm{SO}$ equivalent; $\mathrm{EP}-\mathrm{kg} \mathrm{PO}_{4}$ equivalent; $\mathrm{EU}$ - GJ/FU. All values are for a functional unit.

Table 7. Global warming potential (GWP), acidification potential (AP) and eutrophication potential (EP) values for each activity in scenario 4.

\begin{tabular}{|c|c|c|c|c|c|c|c|c|c|}
\hline \multirow{6}{*}{ GWP } & Emission & $\begin{array}{c}\text { Animal } \\
\text { managemen } \\
\mathrm{t}\end{array}$ & \begin{tabular}{|c|} 
Water \\
pumping
\end{tabular} & Scalding & $\begin{array}{c}\text { Defeatheri } \\
\text { ng }\end{array}$ & Cutting & Packaging & Freezing & \begin{tabular}{|c|} 
Feed \\
production
\end{tabular} \\
\hline & $\mathrm{CO}_{2}$ & $6.67 \mathrm{E}-03$ & $1.11 \mathrm{E}-11$ & 3.34E-10 & 8.31E-11 & 8.31E-11 & 6.37E-11 & 5.13E-09 & 4.63E-09 \\
\hline & $\mathrm{CH}_{4}$ & $1.44 \mathrm{E}-06$ & $1.49 \mathrm{E}-14$ & $4.50 \mathrm{E}-13$ & $1.12 \mathrm{E}-13$ & $1.12 \mathrm{E}-13$ & 8.57E-14 & $6.90 \mathrm{E}-12$ & $6.23 \mathrm{E}-12$ \\
\hline & $\mathrm{N}_{2} \mathrm{O}$ & 8.74E-06 & 2.62E-14 & $7.91 \mathrm{E}-13$ & $1.97 \mathrm{E}-13$ & $1.97 \mathrm{E}-13$ & $1.51 \mathrm{E}-13$ & $1.21 \mathrm{E}-11$ & $1.10 \mathrm{E}-11$ \\
\hline & Total & 6.68E-03 & $1.11 \mathrm{E}-11$ & $3.36 \mathrm{E}-10$ & 8.34E-11 & 8.34E-11 & 6.39E-11 & $5.15 \mathrm{E}-09$ & $4.65 \mathrm{E}-09$ \\
\hline & $\begin{array}{l}\% \text { of Grand } \\
\text { total }\end{array}$ & 99.9998 & $1.66 \mathrm{E}-07$ & 5.03E-06 & $1.25 \mathrm{E}-06$ & $1.25 \mathrm{E}-06$ & $9.57 \mathrm{E}-07$ & 7.711E-05 & $6.97 \mathrm{E}-05$ \\
\hline \multirow{5}{*}{ AP } & $\mathrm{SO}_{2}$ & $4.00 \mathrm{E}-05$ & $5.00 \mathrm{E}-14$ & $1.51 \mathrm{E}-12$ & $3.75 \mathrm{E}-13$ & $3.75 \mathrm{E}-13$ & $2.88 \mathrm{E}-13$ & 2.32E-11 & 2.09E-11 \\
\hline & $\mathrm{NO}_{\mathrm{X}}$ & 3.73E-06 & $6.24 \mathrm{E}-15$ & $1.89 \mathrm{E}-13$ & $4.69 \mathrm{E}-14$ & 4.69E-14 & $3.60 \mathrm{E}-14$ & $2.90 \mathrm{E}-12$ & $2.63 \mathrm{E}-12$ \\
\hline & $\mathrm{NH}_{3}$ & 7.78E-01 & 0 & 0 & 0 & 0 & 0 & 0 & 0 \\
\hline & Total & 7.78E-01 & $5.62 \mathrm{E}-14$ & $1.70 \mathrm{E}-12$ & 4.22E-13 & 4.22E-13 & 3.24E-13 & 2.61E-11 & $2.35 \mathrm{E}-11$ \\
\hline & $\begin{array}{l}\% \text { of Grand } \\
\text { total }\end{array}$ & 99.9998 & 7.22E-12 & 2.18E-10 & $5.43 \mathrm{E}-11$ & $5.43 \mathrm{E}-11$ & $4.16 \mathrm{E}-11$ & 3.35E-09 & $3.025 \mathrm{E}-09$ \\
\hline \multirow{4}{*}{ EP } & $\mathrm{NO}_{\mathrm{X}}$ & 6.93E-07 & $1.16 \mathrm{E}-15$ & $3.51 \mathrm{E}-14$ & $8.71 \mathrm{E}-15$ & $8.71 \mathrm{E}-15$ & $6.68 \mathrm{E}-15$ & $5.38 \mathrm{E}-13$ & $4.86 \mathrm{E}-13$ \\
\hline & $\mathrm{NH}_{3}$ & $1.37 \mathrm{E}-01$ & 0 & 0 & 0 & 0 & 0 & 0 & 0 \\
\hline & \begin{tabular}{|l|} 
Total \\
\end{tabular} & $1.37 \mathrm{E}-01$ & $1.16 \mathrm{E}-15$ & $3.51 \mathrm{E}-14$ & $8.71 \mathrm{E}-15$ & $8.71 \mathrm{E}-15$ & $6.68 \mathrm{E}-15$ & $5.38 \mathrm{E}-13$ & $4.86 \mathrm{E}-13$ \\
\hline & $\begin{array}{l}\% \text { of Grand } \\
\text { total }\end{array}$ & 100 & $8.49 \mathrm{E}-13$ & $2.57 \mathrm{E}-11$ & $6.38 \mathrm{E}-12$ & $6.38 \mathrm{E}-12$ & $4.89 \mathrm{E}-12$ & $3.94 \mathrm{E}-10$ & $3.56 \mathrm{E}-10$ \\
\hline $\mathrm{EU}$ & & 12.5416 & 0.59649 & 126.516 & 31.4471 & 31.4471 & 24.0555 & 1940.723 & 2.33678 \\
\hline
\end{tabular}

Units: GWP - $\mathrm{kg} \mathrm{CO}_{2}$ equivalent; $\mathrm{AP}-\mathrm{kg} \mathrm{SO}_{2}$ equivalent; $\mathrm{EP}-\mathrm{kg} \mathrm{PO}_{4}$ equivalent; $\mathrm{EU}-\mathrm{GJ} / \mathrm{FU}$. All values are for a functional unit.

\subsubsection{Acidification Potential}

Table 8 shows nearly similar acidification potential values for all the scenarios. This impact category depended mostly on the $\mathrm{NH}_{3}$ emissions from animal management and was due to the similar housing facility and stacking of waste from poultry. $\mathrm{NH}_{3}$ emissions from all scenarios accounted for almost $100 \%$ of the total contribution.

Similar to the GWP, the AP value for Scenario 2 was higher, though minimally. This was because the diesel generators emitted slightly higher amounts of $\mathrm{NO}_{\mathrm{X}}$ and $\mathrm{SO}_{\mathrm{X}}$.

\subsubsection{Eutrophication Potential}

As shown in Table 8, the EP values were also practically the same, with a slightly higher value for Scenario 2 than for others. While all the scenarios had similar $\mathrm{NH}_{3}$ emissions due to waste from similar housing facility, the higher emission of $\mathrm{NO}_{\mathrm{X}}$ in Scenario 2 drove its overall EP value slightly higher. Compared to the air-related impact categories, the EP values for all scenarios were not significantly affected by the national power mix.

Animal management EP value for each scenario accounted for almost $100 \%$ of its respective total EP contribution. The eutrophication values for all the scenarios were driven by the $\mathrm{NH}_{3}$ emissions from animal management.

Table 8. Impact assessment summary.

\begin{tabular}{|l|c|c|c|c|}
\hline Scenario & $\begin{array}{c}\text { Global Warming Potential } \\
\left(\mathrm{kg} \mathrm{CO}_{2} \text { equivalent/FU }\right)\end{array}$ & $\begin{array}{c}\text { Acidification Potential } \\
\left(\mathrm{kg} \mathrm{SO}_{2} \text { equivalent/FU) }\right.\end{array}$ & $\begin{array}{c}\text { Eutrophication Potential } \\
\left(\mathrm{kg} \mathrm{PO}_{4} \text { equivalent/FU }\right)\end{array}$ & $\begin{array}{c}\text { Energy used } \\
(\mathrm{GJ} / \mathrm{FU})\end{array}$ \\
\hline Scenario 1 & $6.675 \mathrm{E}-03$ & $7.783 \mathrm{E}-01$ & $1.366 \mathrm{E}-01$ & 1302.98756 \\
\hline Scenario 2 & $235.7 \mathrm{E}-03$ & $7.798 \mathrm{E}-01$ & $1.367 \mathrm{E}-01$ & 3036.33411 \\
\hline Scenario 3 & $6.675 \mathrm{E}-03$ & $7.783 \mathrm{E}-01$ & $1.366 \mathrm{E}-01$ & 2169.66379 \\
\hline Scenario 4 & $6.675 \mathrm{E}-03$ & $7.783 \mathrm{E}-01$ & $1.366 \mathrm{E}-01$ & 2169.66379 \\
\hline
\end{tabular}




\subsubsection{Energy Use}

Table 8 shows the Energy Use (EU) values of all the four scenarios. The high value in Scenario 2 was driven to a large extent by how much of diesel was consumed. Scenario 1, with an EU value of 1302.9875 GJ/FU, had the least energy impact due to the relatively lower equivalent emission factors for purchased power. The difference in national power mix for Scenario 3 and Scenario 4 showed no significant difference. For all of the scenarios, most of the EU came during the freezing process, where a substantial amount of electricity was consumed due to longer operating hours and power ratings of the blast freezers. It was about $89 \%$ of the total EU contribution in each scenario. Scalding process, which involved the use of boilers, contributed only about $6 \%$ of the total EU contribution in each scenario.

\section{CONCLUSIONS}

As mentioned, the electricity production from diesel generators has been responsible for a considerable portion of total GHG emissions. There are numerous technological solutions which offer substantial $\mathrm{CO}_{2}$ reduction potentials, including renewable energies, higher efficiency power generation, fossil-fuel use with $\mathrm{CO}_{2}$ capture and storage, fusion energy, nuclear fission, hydrogen, fuel cells, biofuels, and efficient energy end use. No single technology can meet this challenge by itself. Different regions and countries will need different combinations of technologies to best serve their needs and best exploit their indigenous resources [20]. Nigeria's power systems presently depend on hydropower and thermal power majorly, but power systems of the future must rely on a mix of different advanced, clean, efficient technologies. Fossil and non-fossil energy will be needed in the likely future to meet national energy demands.

With the present state of power generation in Nigeria, coupled with its erratic supply to consumers, most agricultural establishments rely on diesel generators either as back-up or their main sources of electricity. Hence in terms of energy use, reliance on hydropower plants instead of the thermal plants that are run on natural gas impacted minimally on the environmental load. Hence, Power Holding Company of Nigeria (PHCN) advocacy for shift from hydro to thermal plants should be considered with caution in terms global environmental impacts. Thus, to reduce environmental impacts, various emission control technologies including high efficiency diesel particulate filters, flow through filters, diesel extraction catalysts, selective catalysts, reduction, $\mathrm{NO}_{\mathrm{x}}$ absorbers etc., could be used. These technologies could reduce emissions by about $80-90 \%$.

Major contributor to waste load was animal management which included the housing facility for raising layers and for temporary keeping of spent layers. According to [21], $\mathrm{NH}_{3}$ reduction can be done using acidified biochar. This reduces $\mathrm{NH}_{3}$ gases by about 58-63\%.

The efficiency of all production processes can be improved by technology development and good housekeeping practices. For example, stunning live birds before killing them reduces the overall blood loss from splashes. Fullscale poultry slaughterhouse wastewater treatment could be used to remove organic matter as reported by [22] and fully automated slaughtering machine could improve the efficiency frozen chicken production. Energy efficient equipment (e.g. fluorescent lamps) could be used instead of incandescent bulbs, transportation vehicles could be well maintained, and efficient water heaters, freezers and conditioners could be used to minimize power and fuel consumption. Economical use of water could be ensured by avoiding overflows and leaks.

\section{ACKNOWLEDGEMENTS}

The financial support granted lead author by the Netherlands Fellowship Program (NFP) for attending the 2010 Short Course on Cleaner production and the water cycle at UNESCO-IHE Institute for Water Education, Delft Netherlands is appreciated.

\section{REFERENCES}

[1] Food and Agriculture Organization. "Livestock's long shadow" - environmental issues and options. Food and Agricultural Organization of the United Nations, Rome, 2006, p. 390. 
[2] Da Alvarenga, F., da Silva, V.P., Soares, R., Comparison of the ecological footprint and a Life Cycle Impact Assessment method for a case study on Brazilian broiler feed production. Journal of cleaner production, vol. 28, 2012, p. $25-32$.

[3] Farmery, A., Gardner, C., Green, B.S., Jennings, S., Watson, R., Life Cycle Assessment of wild capture Prawns: Expanding sustainability considerations in the Australian Northern Prawn Fishery, Journal of cleaner production, vol. 87, 2015, p. $96-104$.

[4] Lundie, S., Peters, G. M., Life Cycle Assessment of food waste management options, Journal of cleaner production, vol. 13, 2005, p. 275 - 286.

[5] Boggia, A., Paolotti, L., Castellini, C., Environmental impact evaluation of conventional, organic and organicplus poultry production system using life cycle assessment, World's Poultry Science Journal, vol. 66, 2010, p. 95 $-114$.

[6] Hanh, N.T., Bouvarel, I., Ponchant, P., van der Werf, H.M.G., Using environmental constraints to formulate low-impact poultry feeds, Journal of cleaner production, vol. 28, 2012, p. $215-224$.

[7] Ruviaro, C.F., Gianezini, M., Brandao, F.S., Winck, C.A., Dewes, H., Life Cycle Assessment in Brazilian agriculture facing worldwide trends. Journal of Cleaner Production, vol. 28, 2012, p. 9 - 24.

[8] Ewemoje, T.A., Oluwaniyi, O.O., Mechanised Shea butter production in South-western Nigeria using Life Cycle Assessments (LCA) approach from gate-to-gate, AgricEngrgInt: CIGR Journal, vol. 18, no. 2, 2016, p. 230 $-242$.

[9] International Standards Organization (ISO) 14044 Environmental management - Life cycle assessment Requirements and guidelines, International Standards Organization, Brussels, 2006.

[10] Koskela, M., Expert views on environmental impacts and their measurement in the forest industry, Journal of Cleaner Production, vol. 19, 2011, p. 1365 - 1376.

[11] Seiffert, M.E.B., Environmental impact evaluation using a cooperative model for implementing EMS (ISO 14001) in small and medium-sized enterprises, Journal of Cleaner Production, vol. 16, 2008, p. 1447 - 1461.

[12] Ogino A., Orito H., Shimada K., Hirooka K., Evaluating environmental impacts of the Japanese beef CowCalf system by the Life Cycle Assessment method, Journal of Animal Science, vol. 78, 2007, p. 424 - 432.

[13] Biswas, W.K., Barton, L., Carter, D., Global warming potential of wheat production in western Australia: A Life Cycle Assessment, Water and Environment Journal, vol. 22, 2008, p. 206 - 216.

[14] Government of Alberta, Agriculture and rural development: Decision making tools, Ammonia estimator, 2011, http://www.agric.gov.ab.ca/app19/ (12.10.2012)

[15] Hydro-Quebec., Greenhouse gas emissions and reservoirs, 2011, http://www.hydroquebec.com/sustainabledevelopment/documentation/ges.html (20.04.2011)

[16] Leonardo Academy Inc. Emission factors and energy prices for Leonardo academy's cleaner and greener program, 2009, www.leonardoacademy.org (20.04.2011)

[17] Intergovernmental Panel on Climate Change., Climate change 2007: The Physical Science Basis, Cambridge University Press, Cambridge, United Kingdom and New York, NY, USA, 2007.

[18] Azapagic, A., Polymers, the environment and sustainable development, John Wiley \& Sons, ISBN 0-47187741-7, 2003.

[19] Azapagic, A., Perdan, S., Clift, R., Sustainable development in practice - case studies for Engineers and Scientists, John Wiley \& Sons, ISBN 0-470-85609-2, 2004.

[20] International Energy Agency., Energy Technology: Facing the climate challenge, IEA Governing Board, 2003.

[21] Doydora, S.A., Cabrera, L.M., Das, C.K., Gaskin, W.J., Sonon, S.L., Miller, P.W., Release of Nitrogen and Phosphorus from poultry litter amended with acidified biochar, International Journal of Environmental Research and Public Health, vol. 8, 2011, p. 1491 - 1500.

[22] Del Nery, V., De Nardi, I., Damianovic, M., Pozzi, E., Amorim, A., Zaiat, M., Long-time operating performance of a poultry slaughterhouse wastewater treatment plant, Resources conservation and recycling, vol. 50, Issue 1, 2007, p. $102-114$. 